\title{
ORDER TYPES AND SIMILARITY TRANSFORMATIONS ${ }^{1}$
}

\author{
BY \\ SEYMOUR GINSBURG
}

Suppose that $\sigma$ and $\mu$ are two distinct order types for which $\sigma<\mu\left({ }^{2}\right)$. The question of the existence of an order type $\tau$ for which $\sigma<\tau<\mu$ is hereafter referred to as "problem P." Whenever such an order type $\tau$ exists we shall say problem $\mathrm{P}$ admits of a solution. The two purposes of this paper are

(a) to study the order types of simply ordered sets of solutions to problem $\mathrm{P}$, as applied to the cases where (I) $\sigma$ is any linear order type and $\mu=\lambda\left(^{3}\right)$, and (II) $\sigma=0, \mu$ is any linear order type of power $2^{\aleph_{0}}$, and $\tau$ is of power $2^{\aleph_{0}}$; and (b) to study pairs of simply ordered sets whose symmetric difference contains just two elements and whose order types are incomparable.

\section{Exact sets.}

Definition. A simply ordered set $E$ is called exact if $E$ is similar to no proper subset of itself.

If $E$ is exact, then the order type of $E$ is called exact.

It is easily seen that $E$ is exact if and only if each point of $E$ is a fixed point of $E\left({ }^{4}\right)$. It is also easily seen that if $B$ is exact and $A \subset B$, then $\bar{A}<\bar{B}$.

The following two facts on exact sets are known [1]:

A. There does not exist an exact set of $\boldsymbol{\aleph}_{0}$ elements.

B. There exists a dense subset of $R$, of power $2^{\aleph_{0}}$, which is exact.

The following result is immediately obtained from (I) of $\$ 2$ of [3].

THEOREM 1. If $E$ is exact and $\bar{F} \equiv \bar{E}$, then there is one and only one similarity transformation $g$ of $E$ into $F$. Furthermore, $g(E)=F$.

Corollary. If $E$ is exact and $\bar{E} \equiv \bar{F}$, then $F$ is exact. Furthermore, $\bar{E}=\bar{F}$.

Presented to the Society, February 28, 1953, under the title Incomparable order types and September 5, 1953, under the title of Order types of simply ordered sets of solutions to problem $\mathrm{P}$; received by the editors May 28, 1954.

(1) In three previous papers $[3 ; 4 ; 5]$ on order types, the author has made numerous references to an article which had been submitted to another journal. Since that paper is not to appear in the designated publication, its results, together with other material, have been coalesced to form the present paper.

(2) Let $A$ and $B$ be two simply ordered sets, with $\bar{A}$ and $\bar{B}$ being the order types of $A$ and $B$ respectively. Let $\bar{A} \leqq \bar{B}$ if there exists a similarity transformation of $A$ into $B$. Let $\bar{A} \equiv \bar{B}$ if there is a similarity transformation of $A$ into $B$ and a similarity transformation of $B$ into $A$. $\bar{A}$ and $\bar{B}$ are incomparable order types, denoted by $\bar{A} \| \bar{B}$, if there is no similarity transformation of $A$ into $B$ and no similarity transformation of $B$ into $A$. If $\bar{A} \equiv \bar{B}$ is false, then $\bar{A}$ and $\bar{B}$ are called distinct. Let $\bar{A}<\bar{B}$ if $\bar{A} \leqq \bar{B}$, but $\bar{A}$ and $\bar{B}$ are distinct. See [11].

(3) By $R$ is meant the set of real numbers, ordered in the natural manner. By $\lambda$ is meant the order type of $R$. By linear set is meant any subset of $R$.

$\left(^{4}\right) p$ is a fixed point of $E$ if $f(p)=p$ for each similarity transformation $f$ of $E$ into $E$. 
We now consider some other properties of exact order types.

TheOREM 2. If $\alpha$ and $\beta$ are exact order types, then so is $\alpha+\beta$.

Proof. Let $A$ and $B$ be two sets such that $\bar{A}=\alpha$ and $\bar{B}=\beta$ and such that $A+B$ is an ordered sum( ${ }^{(5)}$. Let $f$ be a similarity transformation of $A \cup B$ into a proper subset of $A \cup B$. Suppose that $f(A)$ is a proper subset of $A$. Then this contradicts the fact that $A$ is exact. Therefore $f(B)$ is a proper subset of $B$. But this contradicts $B$ being exact. Therefore no such similarity transformation can exist, i.e., $\bar{A}+\bar{B}$ is exact.

Since a finite ordinal number is exact we get

CoROLlary 1. If $\alpha$ is exact and $n$ is a finite ordinal number, then $n+\alpha$ and $\alpha+n$ are both exact.

It is easily seen that the converse to Theorem 2 is also true, i.e., if $\gamma$ is exact and $\gamma=\alpha+\beta$, then both $\alpha$ and $\beta$ are exact. From this we infer

Corollary 2. If $A$ is exact and $B$ is a finite set, then $A \cup B$ and $A-B$ are both exact.

From Theorem 2.2 of [3] and Corollary 2 above we get

THEOREM 3. Let $\gamma=\alpha+\beta$ be exact. Then problem $\mathrm{P}$, as applied to $\sigma=\gamma$ and $\mu=\alpha+n+1+\beta$, admits of precisely $n$ distinct solutions. These are $\tau=\alpha+j+1$ $+\beta$, where $j<n$. Problem $\mathrm{P}$, as applied to $\sigma=\gamma$ and $\mu=\alpha+\omega+\beta$, admits of precisely $\boldsymbol{\aleph}_{0}$ distinct solutions. These are $\tau=\alpha+n+1+\beta$, where $n<\omega$.

In preparation for Theorem 4 we state Lemma 1. The proof of this result follows from the well known Cantor-Meray process for completing a space.

Lemma 1. Any simply ordered, dense set $B\left({ }^{6}\right)$, with no first and no last element, is similar to a set $A$ which is topologically dense in $R$.

COROLlary. Let $B$ be a nonempty simply ordered set such that each point of $B$ is a two sided limit point of $B$. Then $B$ is dense and thus similar to a topologically dense subset of $R$.

We shall call $p$ a right sided (left sided) $c$-condensation point of the linear set $E$ if, for each real number $y$, where $y<p(p<y)$, the open interval $(y, p)$ $((p, y))$ of $R$ contains $2^{\aleph_{0}}$ elements of $E$. A point which is both a right sided and a left sided $c$-condensation point of $E$ shall be called a $c$-condensation point of $E$.

(5) By the ordered sum of the family $\left\{E_{a} \mid a \in A\right\}$ of pairwise disjoint sets, where $A$ is simply ordered, is meant the set $C=\bigcup_{a \in A} E_{a}$, the elements being ordered as follows. If $u<v$ in $E_{a}$, then $u<v$ in $C$. If $u$ is in $E_{a}$ and $v$ is in $E_{b}$, where $a<b$, then $u<v$.

(6) A simply ordered set $E$ is dense if, for each pair of elements $x$ and $y$ in $E, x<y$, a third element $z$ in $E$ can be found so that $x<z<y$. 
A linear set, of power $2^{\aleph_{0}}$, is said to have property $C$ if each point of $E$ is a $c$-condensation point of $E$.

Turning to problem $\mathrm{P}$ we have

Theorem 4. Let $E$ be a linear set of power $2^{N_{0}}$. Then problem $\mathrm{P}$, as applied to $\sigma=0$ and $\mu=\bar{E}$, admits of a solution $\tau$, of power $2^{\kappa_{0}}$, which is exact.

Proof. Let $B$ be the set of $c$-condensation points of $E$ which are in $E$. The set $B$ satisfies the hypotheses of the corollary to Lemma 1 . Hence there exists a similarity transformation $h$ of $B$ onto a dense subset $G$ of $R$, each point of $G$ being a $c$-condensation point of $G$. If $A$ is an exact subset of $G$, of power $2 \aleph_{0}$, then by Corollary 2 of Theorem $2, A-\{p\}$ is exact, $p$ being a point of $A$. Since $A$ is exact, $\overline{A-\{p\}}<\bar{A}$. Then $\tau=\overline{A-\{p\}}$ will satisfy the conclusion of the theorem. The construction of an exact subset of $G$, of power $2 \aleph^{N_{0}}$, has been given in several places already, for example Theorem 1.1 of [4]. In each case the proof has been quite complicated since other properties were demanded. Accordingly, we now present a simple proof that $G$ contains an exact subset of power $2^{\aleph_{0}}$.

Denote by $K^{*}(G)$ the set of those similarity transformations of $G$ into $R$ which are not the identity transformation. Well order the elements of $R$ and $K^{*}(G)$ into the two sequences $\left\{x_{\xi}\right\}, \xi<\theta$, and $\left\{f_{\xi}\right\}, \xi<\theta\left({ }^{7}\right)$, respectively. By transfinite induction we define a subset of $G$ as follows:

Let $u_{0}$ be the first element for which $f_{0}(x) \neq x$ and let $v_{0}=f_{0}\left(u_{0}\right)$. Since $f_{0}$ is not the identity transformation of $G$ the element $u_{0}$ certainly exists. Suppose that $u_{\xi}$ and $v_{\xi}$ have been defined for $\xi<\alpha<\theta$. Since $f_{\alpha}$ is a similarity transformation of $G$, the set of those points $x$ for which $f_{\alpha}(x)=x$ cannot be dense in $G$. Thus there exist two real numbers, $a_{\alpha}$ and $b_{\alpha}$, where $a_{\alpha}<b_{\alpha}$, such that $f_{\alpha}(x) \neq x$ for $x$ in $H_{\alpha}, H_{\alpha}$ being defined as the set $G \bigcap\left\{y \mid a_{\alpha}<y<b_{\alpha}\right\}$. Since each element in $G$ is a $c$-condensation point of $G$, the power of $H_{\alpha}$ is $2^{\aleph_{0}}$. Now the power of the set

$$
S_{\alpha}=\left\{u_{\xi} \mid \xi<\alpha\right\} \cup\left\{v_{\xi} \mid \xi<\alpha\right\}
$$

is smaller than $2 \aleph_{0}$. Hence there exists an element $x$ in the set $H_{\alpha}-\left[S_{\alpha}\right.$ $\left.\cup f_{\alpha}^{-1}\left(S_{\alpha}\right)\right]\left({ }^{8}\right)$. Let $u_{\alpha}$ be the first such element, and $v_{\alpha}=f_{\alpha}\left(u_{\alpha}\right)$. Let $A=\left\{u_{\xi} \mid \xi<\theta\right\}$.

If $J$ is any interval in $R$, then there exists a similarity transformation of $G$ into $R$ which is the identity on $G-J$ and is not the identity on $J \cap G$. Thus there exists an ordinal number $\alpha$ for which $u_{\alpha}$ is in $G \cap J$, i.e., $A$ is a dense subset of $G$.

It now follows that the set $A$ is exact. For if $f$ is a similarity transformation of $A$ into $R$ which is not the identity, then $f$ can be extended to be an element $f_{\alpha}$ of $K^{*}(G)$. Therefore the element $f\left(u_{\alpha}\right)=f_{\alpha}\left(u_{\alpha}\right)$ is not in $A$. Thus

(7) By $\theta$ is meant the smallest ordinal number whose power is $2^{\aleph_{0}}$.

(8) By $f^{-1}$ is meant the inverse function of $f$. 
there is no similarity transformation of $A$ into itself which is not the identity, i.e., $A$ is exact.

\section{Property A.}

Definition. A linear set $E$ is said to have property $\mathrm{A}$ if the power of $E$ is $2^{\aleph_{0}}$, and no two disjoint subsets of $E$, of power $2^{\aleph_{0}}$ each, are similar. If $E$ has property $\mathrm{A}$, then $\bar{E}$ is said to have property $\mathrm{A}$.

The existence of a set having property $A$ was first shown in $[2$, pp. 609$610]$ and [11, pp. 263-264]. We shall show that if $E$ is any linear set of power $2^{\aleph_{0}}$, then problem $\mathrm{P}$, as applied to $\sigma=0$ and $\mu=\bar{E}$, admits of a solution $\tau$ having property $A$. The proof differs from that given in [2] and [11].

Lemma 2. Let $E$ be a linear set and $f$ a similarity transformation of $E$ into $R$. Then $f$ can be extended to be a similarity transformation into $R$ of some Borel set $B$ which contains $E$.

Proof. Let $E^{*}$ be the topological closure of $E$, and $A$ the set of one-sided limit points of $E^{*}$ which do not belong to $E$. Now $A$ is enumerable [6, p. 171]. Therefore, if $B=E^{*}-A$, then $B$ is a Borel set which contains $E$. Let $g$ be the function which is defined by $g(y)=f(y)$ for $y$ in $E$, and $g(y)=\lim _{x \rightarrow y} f(x)$ for $y$ in $B-E$.

Let $u$ and $v$, where $u<v$, be any two elements of $B$. Clearly $g(u) \leqq g(v)$. If $u$ and $v$ are each elements of $E$, then $g(u)<g(v)$. If at least one of the two points is in $B-E$, then since it is a two-sided limit point of $E^{*}$ and thus of $E$, it follows that there exist two points, $x$ and $y$, of $E$, such that $u<x<y<v$. Then

$$
g(u) \leqq g(x)<g(y) \leqq g(v) .
$$

Thus $g$ is a similarity transformation of $B$ into $R$.

THEOREM 5. Let $E$ be any linear set of power $2 \aleph^{\aleph_{0}}$. For $\sigma=0$ and $\mu=\bar{E}$, problem $\mathrm{P}$ admits of a solution $\tau$ which has property $\mathrm{A}$.

Proof. By Theorem 1 of [11], there exists a subset $Q$ of $E$, of power $2 \mathrm{~N}_{0}$, such that $\bar{Q}<\bar{E}$. It will be sufficient to show that $Q$ contains a set which has property $\mathrm{A}$.

Denote by $V$ the set of those Borel sets $B$ in $R$ for which the power of the set $B \cap Q$ is $2^{\aleph_{0}}$. For each element $B$ in $V$ let $L(B)$ be the set of those similarity transformations $f$ of $B$ into $R$ for which the power of the set $\{x \mid f(x) \neq x$, $x \in B \cap Q\}$ is $2^{N_{0}}$. Denote by $S$ the set of couples,

$$
S=\{(B, f) \mid B \in V, f \in L(B)\} .
$$

The power of the set $S$ is $2 \aleph_{0}$. Well order the elements of $Q$ and $S$ into the two sequences $\left\{x_{\xi}\right\}, \xi<\theta$, and $\left\{s_{\xi}\right\}, \xi<\theta$, where $s_{\xi}=\left(B_{\xi}, f_{\xi}\right)$. For each function $f_{\xi}$ define $J_{\xi}$ to be the set

$$
J_{\xi}\left\{x \mid f_{\xi}(x) \neq x, x \in B_{\xi} \cap Q\right\} .
$$


Denote by $p_{0}$ the first element in $J_{0}$. Now suppose that the element $p_{\xi}$ has been defined for each $\xi<\alpha$, where $\alpha<\theta$. I.et $P_{\alpha}=\left\{p_{\xi} \mid \xi<\alpha\right\}$, and $p_{\alpha}$ be the first element in the set $J_{\alpha}-D_{\alpha}$, where

$$
D_{\alpha}=P_{\alpha} \cup \underset{\xi<\alpha}{\cup} f_{\xi}\left(P_{\alpha}\right) \cup \underset{\xi<\alpha}{\bigcup} f_{\xi}^{-1}\left(P_{\alpha}\right) .
$$

The element $p_{\alpha}$ certainly exists since the power of $J_{\alpha}$ is $2^{N_{0}}$ and the power of $D_{\alpha}$ is $\left\langle 2 \aleph_{0}\right.$.

Denote by $M$ the set $M=\left\{p_{\xi} \mid \xi<\theta\right\}$. Suppose that $F$ and $G$ are two disjoint subsets of $M$, of power $2^{N_{0}}$ each, and that $f$ is a similarity transformation of $F$ onto $G$. By Lemma 2, $f$ may be extended to become an element of $L\left(B_{\xi}\right)$ for some ordinal number $\xi$, where $B_{\xi}$ is an element of $V$ which contains the set $F$. For each element $p_{\alpha}$ in $F$ consider the element $f_{\xi}\left(p_{\alpha}\right)$, where $\xi<\alpha$. As $f_{\xi}$ maps $F$ onto $G, f_{\xi}\left(p_{\alpha}\right) \neq p_{\alpha}$. For each ordinal number $\delta>0, p_{\alpha+\delta}$ is not an element of the set $f_{\xi}\left(P_{\alpha+\delta}\right)$. Since $p_{\alpha}$ is in $P_{\alpha+\delta}, f_{\xi}\left(p_{\alpha}\right) \neq p_{\alpha+\delta}$. Suppose that $f_{\xi}\left(p_{\alpha}\right)=p_{\gamma}$, where $\gamma<\alpha$. Then $f_{\xi}\left(p_{\alpha}\right)$ is an element of $P_{\alpha}$, so that $p_{\alpha}$ is an element of $f_{\xi}^{-1}\left(P_{\alpha}\right)$. But this contradicts the manner in which the element $p_{\alpha}$ was selected. Thus $f_{\xi}\left(p_{\alpha}\right) \neq p_{\gamma}$ for $\gamma<\alpha$. Therefore, for $p_{\alpha}$ in $F, f_{\xi}\left(p_{\alpha}\right)$ is not an element of $M$ if $\xi<\alpha$. Consequently the power of the set $f_{\xi}(F) \cap M$ must be $<2^{\aleph_{0}}$. But $f_{\xi}(F) \cap M=G \cap M=G$, and the power of $G$ is $2^{\aleph_{0}}$. From this contradiction, we see that the two sets $F$ and $G$ cannot exist, i.e., the set $M$ has property A. Q.E.D.

REMARK. The above demonstration furnishes yet another proof of Theorem 1 of [11]. To see this define $Q$ to be the set of $c$-condensation points of $E$ which are in $E$, and obtain $M$ as in the theorem. Let $M$ be the union of two disjoint subsets $T$ and $U$, of power $2^{\aleph_{0}}$ each. The set $T$ has property $\mathrm{A}$, and as is easy to see, $\bar{T}<\bar{Q}<\bar{E}$.

3. Order types of simply ordered sets of solutions to problem $P$. The following known result will be used: If $E$ has properties $\mathrm{A}$ and $\mathrm{C}$, then $E$ is exact [3].

Suppose that $\sigma$ is any order type which is $<\lambda$. By Theorem 2.4 of [3], or by the remark at the end of $\S 2$, there exists an exact subset $E$ of the open interval $(0,1)$ for which $\sigma<\bar{E}$. Let $X$ be a subset of the open interval $(1,2)$ which has properties $\mathrm{A}$ and $\mathrm{C}$. It is well known that $X$ is the union of two disjoint sets $U$ and $V$, each having property $\mathrm{C}$ and each dense in $X$. Let $K$ be any subset of $V$. The set $U \cup K$ has properties $\mathrm{A}$ and $\mathrm{C}$, and hence is exact. Therefore, by Theorem 2, the set $E \cup U \cup K$ is exact. From this we see that if $K$ and $L$ are any two subsets of $V$ such that $K \subset L$, then

$$
\bar{E} \cup \bar{U} \bar{U}<\bar{E} \bar{U} \bar{L} \text {. }
$$

Now let $Y$ be any abstract set of power $2^{\aleph_{0}}$, and $H$ a simply ordered family of subsets of $Y$. Since $Y$ and $V$ have the same power, there exists a one to one 
function of $Y$ onto $V$. From the discussion it follows that the family $H$ is similar to the set of order types

$$
\{\bar{P} \mid P=E \cup U \cup f(M), M \in H\}
$$

under the mapping which takes $M$ into $\overline{E \cup U \cup f(M)}$. This implies that problem $\mathrm{P}$, as applied to any order type $\sigma$ which is $\langle\lambda$, and $\mu=\lambda$, has a simply ordered set, which is similar to $H$, of exact solutions, each solution being of power $2^{\aleph_{0}}$. While not used in the sequel, it is true that the mapping which takes $M$ into $\overline{E \cup U \cup f(M)}$ has the following property:

The mapping is a one to one, order preserving function, of the family $\{M \mid M \subseteq Y\}$ onto the set $\{\tau \mid \overline{E \cup U} \leqq \tau \leqq \overline{E \cup U \cup V}\}$. Furthermore, the inverse function is also order preserving.

To see this it is sufficient to show that if $\tau$ is any order type for which $\overline{E \cup U}<\tau<\overline{E \cup U \cup V}$, then there exists a subset $K$ of $V$ such that $\tau=\overline{E \cup U \cup K}$. Let $F$ be a subset of $E \cup U \cup V$ such that $\bar{F}=\tau$. Let $B$ and $W$ be two subsets of $F$, with $\bar{E}=\bar{B}$ and $\bar{U}=\bar{W}$, such that $B \cup W$ is an ordered sum. Hence $B \cup W$ is a subset of $E \cup U \cup V$. First suppose that $B$ is a subset of $E$. Since $E$ is exact and $\bar{B}=\bar{E}$, it follows that $B=E$. This implies that $W$ is a subset of $U \cup V$. Now the set $U \cup V$ has properties $A$ and $C$, while $W$ has the property that for any two elements $a$ and $b$ in $W$, the power of the set $\{x \mid a<x<b, x \in W\}$ is $2^{\aleph_{0}}$. From this we see that each element of $W$ must be a fixed $(W, U \cup V)$ point $\left({ }^{9}\right)$. As $\bar{W}=\bar{U}$, it follows that $W=U$. Consequently, for some subset $K$ of $V, F=E \cup U \cup K$. Suppose, on the other hand, that $B$ is not a subset of $E$. Then $W$ is a subset of $U \cup V$. As above we see that $W=U$. Therefore $B$ is a subset of $E$. From this we see that $F=E \cup U \cup K$ for some subset $K$ of $V$.

Now let $\alpha$ be any linear order type of power $2^{\aleph_{0}}$. Let $D$ be a linear set whose order type is $\alpha$. $D$ contains a set $E$ which has property A, by Theorem 5. Let $X$ be the set of $c$-condensation points of $E$ which are in $E$. The set $X$ has properties $\mathrm{A}$ and $\mathrm{C}$. Repeating the procedure given above we see that the mapping which takes $M$ into $\overline{U \cup f(M)}$ is a one to one, order preserving function, of the family $\{M \mid M \subseteq Y\}$ onto the set $\{\tau \mid \bar{U} \leqq \tau \leqq \overline{U \cup V}\}$. Furthermore, the inverse function is also order preserving. From this we see that problem $\mathrm{P}$, as applied to $\sigma=0$ and $\mu=\alpha$, has a set, which is similar to $H$, of exact solutions, each solution being of power $2 \aleph^{\aleph_{0}}$.

Summarizing the preceding discussion we have the following two results:

THEOREM 6. Let $\alpha$ be any order type which is $<\lambda$. Let $Y$ be any set of power $2 \aleph_{0}$ and $H$ a simply ordered family of subsets of $Y$, ordered by set inclusion. Then problem $\mathrm{P}$, as applied to $\sigma=\alpha$ and $\mu=\lambda$, admits of a set, which is similar to $H$, of exact solutions, each solution being of power $2 \mathrm{~N}_{0}$.

THEOREM 7. Let $\alpha$ be any linear order type of power $2^{\aleph_{0}}$. Let $Y$ be any set of power $2^{\aleph_{0}}$ and $H$ a simply ordered family of subsets of $Y$, ordered by set in-

(9) See $\$ 4$. 
clusion. Then problem $\mathrm{P}$, as applied to $\sigma=0$ and $\mu=\alpha$, admits of a set, which is similar to $H$, of exact solutions, each solution being of power $2^{N_{0}}$.

Theorems 6 and 7 yield several consequences. For example, let $Y=R$ and let $S$ be the set of rational numbers ordered in the natural manner. Denote by $H$ the family of sets

$$
H=\left\{E_{x} \mid x \in R ; E_{x}=\{y \mid y<x, y \in S\}\right\} .
$$

The order type of $H$ is $\lambda$. Consequently we obtain

THEOREM 8. Let $\alpha$ be any order type which is $<\lambda$. There exist two linear sets $E$ and $F$, where $E \subset F$ and $F-E$ is denumerably infinite, with the following two properties: (1) $\alpha<\bar{E}<\bar{F}<\lambda$; and (2) problem $\mathrm{P}$, as applied to $\sigma=\bar{E}$ and $\mu=\bar{F}$ admits of a set, of order type $\lambda$, of exact solutions, each solution being of power $2^{\aleph_{0}}$.

Corollary. Let $\alpha$ be any order type which is $<\lambda$. For each linear order type $\beta$, problem $\mathrm{P}$, as applied to $\sigma=\alpha$ and $\mu=\lambda$, admits of a set, of order type $\beta$, of exact solutions, each solution being of power $2^{\aleph_{0}}$.

THEOREM 9. Let $\alpha$ be any linear order type of power $2^{\aleph_{0}}$. There exist two linear sets $E$ and $F$, where $E \subset F$ and $F-E$ is denumerably infinite, with the following two properties: (1) $\bar{E}<\bar{F}<\alpha$, and (2) problem $\mathrm{P}$, as applied to $\sigma=\bar{E}$ and $\mu=\bar{F}$, admits of a set, of order type $\lambda$, of exact solutions, each solution being of power $2^{\kappa_{0}}$.

Conollary. Let $\alpha$ and $\beta$ be any two linear order types, the power of $\alpha$ being $2 \aleph_{0}$. Then problem $\mathrm{P}$, as applied to $\sigma=0$ and $\mu=\alpha$, admits of a set, of order type $\beta$, of exact solutions, each solution being of power $2^{\aleph_{0}}$.

REMARK. Theorems 3 and 4 state that for any linear order type $\alpha$, of power $2 \aleph_{0}$, there exist two linear sets $E$ and $F$, where $E \subset F$ and $F-E$ is denumerably infinite, with the following two properties: (1) $\bar{E}<\bar{F}<\alpha$, and (2) problem $\mathrm{P}$, as applied to $\sigma=\bar{E}$ and $\mu=\bar{F}$, admits of a set $T$, of order type $\omega$, of exact solutions, each solution being of power $2^{\aleph_{0}}$. The set $F-E$ obtained consists of $\boldsymbol{\aleph}_{0}$ elements, no one of the elements being a fixed point of $F$. In Theorem 9 the set $F-E$ contains $\boldsymbol{\aleph}_{0}$ elements, each of which is a fixed point of $F$.

Using Theorem 2.4 of [3], a similar discussion is possible for two sets $E$ and $F$ for which $\alpha<\bar{E}<\bar{F}<\lambda$.

Sierpiński $[9 ; 10]$ has shown that if $Y$ is a set of power $2^{\aleph_{0}}$, then there exists a simply ordered family, of power $>2 \aleph^{\aleph_{0}}$, of subsets of $Y$, ordered by set inclusion. This in conjunction with Theorems 6 and 7 yields

TheOREM 10. Let $\alpha$ be any order type which is $<\lambda$. Then problem $\mathrm{P}$, as applied to $\sigma=\alpha$ and $\mu=\lambda$, admits of a simply ordered set, of power $>2^{\aleph_{0}}$, of exact solutions, each solution being of power $2^{\aleph_{0}}$. 
TheOREM 11. Let $\alpha$ be any linear order type of power $2^{\aleph_{0}}$. Then problem $\mathrm{P}$, as applied to $\sigma=0$ and $\mu=\alpha$, admits of a simply ordered set, of power $>2 \aleph_{0}$, of exact solutions, each solution being of power $2{ }^{\aleph_{0}}$.

Another proof of Theorem 10, without appealing to Theorem 6, will be given later (Corollary to Theorem 15).

We now consider "decreasing" sequences of solutions to problem $\mathrm{P}$.

THEOREM 12. Let $E$ be any linear set of power $2^{\mathrm{N}_{0}}$ and $H$ a simply ordered set of power $\leqq 2^{\aleph_{0}}$. Then problem $\mathrm{P}$, as applied to $\sigma=0$ and $\mu=\bar{E}$, admits of $a$ set $T$, of order type $\bar{H}$, of exact solutions, of power $2 \aleph_{0}$ each, so that there is no order type $\tau$, of power $2^{\aleph_{0}}$, which is $<\beta$ for each $\beta$ in $T$, if and only if $H$ has no first element.

Proof. Suppose that $H$ has no first element. Let $B$ be any subset of $E$ having properties $A$ and $C$. Decompose $B$ into a family of disjoint sets $\left\{C_{h} \mid h \in H\right\}$, where each set $C_{h}$ has property $\mathrm{C}$ and is also topologically dense in $B$. An obvious modification in the construction of the sets $\left\{C_{\xi}\right\}$ defined in Theorem 3.4 of [4], which is possible since the power of $H$ is $\leqq 2 \aleph_{0}$, shows that such a family of sets exists. Let $E_{h_{0}}=B-\bigcup_{h \geqq h_{0}} C_{h}$ for each $h_{0}$ in $H$. Clearly each set $E_{h}$ has properties A and C. Since $E_{h_{0}} \subset E_{h_{1}}$ for $h_{0}<h_{1}$, the set of order types $T=\left\{\bar{E}_{h} \mid h \in H\right\}$ is similar to $H$. Thus since $H$ has no first element, $\bigcap_{h \in H} E_{h}=\varnothing$.

We now show that there is no order type $\tau$, of power $2^{\aleph_{0}}$, which is $\left\langle\bar{E}_{h}\right.$ for each $h$. Assume the contrary, i.e., suppose that $\tau$ is an order type, of power $2^{\aleph_{0}}$, which is $\left\langle\bar{E}_{h}\right.$ for each $h$. Let $g$ be a definite element of $H$. Let $G$ be a subset, of order type $\tau$, of $E_{g}$, and $D$ the set of $c$-condensation points of $G$ which are in $G$. The set $D$ has properties A and C. As $E_{g}$ has property A, each element of $D$ is a fixed $\left(D, E_{g}\right)$ point. For each $h<g$ let $F_{h}$ be a subset of $E_{h}$ such that $\bar{F}_{h}=\bar{D}$. The set $F_{h}$ certainly exists since $\bar{F}_{h}<\bar{E}_{h}$. Now $D$ and $F_{h}$ are two similar subsets of $E_{g}$. Since each element of $D$ is a fixed $\left(D, E_{g}\right)$ point it follows that $D=F_{h}$. Therefore $D$ is a subset of each set $E_{h}, h \leqq g$. Then $D \subseteq \bigcap_{h \leqq 0} E_{h}=\bigcap_{h \in H} E_{h}=\varnothing$. But the set $D$ has $2^{\aleph_{0}}$ elements. From this contradiction it follows that no such order type $\tau$ can exist.

Suppose that $H$ has a first element, say $g$. Let $\left\{\alpha_{h}\right\}$ be any set of exact order types, where each $\alpha_{h}$ is of power $2^{\aleph_{0}}$, and $\alpha_{h_{0}}<\alpha_{h_{1}}$ for $h_{0}<h_{1}$ in $H$. By Theorem 4 there exists an exact order type $\tau$, of power $2^{N_{2}}$, satisfying $0<\tau<\alpha_{o} \leqq \alpha_{h}$. Q.E.D.

Corollary. Let $E$ be any linear set of power $2^{\aleph_{0}}$. Let $\gamma$ be any limit number of power $\leqq 2^{\aleph_{0}}$. Then problem $\mathrm{P}$, as applied to $\sigma=0$ and $\mu=\bar{E}$, admits of a set $T$, of order type $\gamma^{*}$, of exact solutions, of power $2^{N_{0}}$ each, with the following property: There is no order type $\tau$, of power $2^{\aleph_{0}}$, such that $\tau<\beta$ for each element $\beta$ in $T$.

The question of whether or not the power of $H$ can be $>2^{\aleph_{0}}$ arises. On assuming that $2 \boldsymbol{\aleph}_{0}=\boldsymbol{\aleph}_{1}$ the answer shall be shown to be in the negative. 
TheORem 13. Let $E$ have property A. If $2^{\aleph_{0}}=\aleph_{1}$ and if $\left\{\alpha_{\xi}\right\}, \xi<\gamma$, is a strictly decreasing sequence of order types, with $\alpha_{0}=\bar{E}$, then $\bar{\gamma} \leqq 2^{\aleph_{0}}$.

Proof. For each $\xi$ let $E_{\xi}$ be a subset, of order type $\alpha_{\xi}$, of $E$. Denote by $D_{\xi}$ the set of $c$-condensation points of $E_{\xi}$ which are in $E_{\xi}$. Suppose that $p$ is an element of $D_{\xi}$. Since $E$ has property $\mathrm{A}, p$ is a fixed $\left(D_{\xi}, E\right)$ point $\left({ }^{10}\right)$. For $v<\xi, \bar{D}_{\xi} \leqq \bar{E}_{\xi}<\bar{E}_{v}$. Hence there exists a similarity transformation $f$ of $D_{\xi}$ into $E_{v}$. Since $E_{v}$ is a subset of $E$ and $p$ is a fixed $\left(D_{\xi}, E\right)$ point, it follows that $f(p)=p$. Thus $p$ is in $E_{v}$, i.e., $D_{\xi}$ is a subset of $E_{v}$. As each element $p$ of $D_{\xi}$ is a $c$-condensation point of $D_{\xi}$, thus of $E_{v}, p$ is also in $D_{v}$. In other words, for $v<\xi, D_{\xi}$ is a subset of $D_{v}$. Thus we have

$$
D_{0} \supseteq D_{1} \supseteq \cdots \supseteq D_{\xi} \supseteq \cdots
$$

Note that since the power of $D_{0}$ is $2 \boldsymbol{N}_{0}$, there are at most $2^{\boldsymbol{N}_{0}}$ different sets $D_{\xi}$.

Assuming that $2 \boldsymbol{\aleph}_{0}=\boldsymbol{\aleph}_{1}$ we see that the power of the set $E_{\xi}-D_{\xi}$ is $\leqq \boldsymbol{\aleph}_{0}$. For a given $\xi$ as $\bar{D}_{\xi}<\lambda$, the power of the set $R-D_{\xi}$ is $2^{N_{0}}$ [11]. There are exactly $\left(2 \boldsymbol{\aleph}_{0}\right) \aleph_{0}=2 \boldsymbol{\aleph}_{0}$ distinct subsets, of power $\leqq \boldsymbol{\aleph}_{0}$ each, of $R-D_{\xi}$. Since $E_{\xi}=D_{\xi} \cup Q_{\xi}$, where $Q_{\xi}$ is a subset of $R-D_{\xi}$ of power $\leqq \boldsymbol{\aleph}_{0}$, it follows that for a given set $D_{\xi}$ there can be at most $2^{\aleph_{0}}$ sets $E_{v}$ which have the property that $D_{v}=D_{\xi}$. This, in conjunction with the fact that there are at most $2 \aleph_{0}$ different sets $D_{\xi}$, implies that $\bar{\gamma} \leqq 2 \aleph_{0}$. Q.E.D.

Suppose that there is no order type $\tau$, of power $2^{\aleph_{0}}$, which is $\langle\alpha \xi$ for each $\xi$. This implies that the power of the set $\bigcap_{\xi<\gamma} D_{\xi}$ is $\left\langle 2^{\aleph_{0}}\right.$. Therefore there is no smallest (as a point set) $D_{\xi}$. Since there are at most $2^{\aleph_{0}}$ different sets $D_{\xi}$, without assuming that $2 \boldsymbol{N}_{0}=\boldsymbol{N}_{1}$ we obtain

TheOrem 14. Let E have property A and let $\left\{\alpha_{\xi}\right\}, \xi<\gamma$, be a strictly decreasing sequence of order types, with $\alpha_{0}=\bar{E}$. If there is no order type $\tau$, of power $2^{\aleph_{0}}$, which is $<\alpha_{\xi}$ for each $\xi$, then there exists an ordinal number $\rho$, where $\rho \leqq \theta$, which is cofinal with $\gamma$.

The problem of whether or not there exists any linear order type $\alpha_{0}$, such that a strictly decreasing sequence of order types $\left\{\alpha_{\xi}\right\}, \xi<\gamma$, where $\bar{\gamma}>2^{\aleph_{0}}$, can be found remains unanswered.

We now turn to strictly increasing sequences of linear order types. In [11] Sierpiński has shown that if $\left\{\alpha_{n}\right\}, n<\omega$, is any sequence of order types, each $\alpha_{n}$ being $<\lambda$, then there exists an order type $\alpha$ such that $\alpha_{n}<\alpha<\lambda$ for each $n$. We now consider an extension of this result. Suppose that $\left\{\alpha_{\xi}\right\}, \xi<\theta$, is a sequence of order types, each $\alpha \xi$ being $<\lambda$. We shall show that there exists an order type $\alpha$ such that $\alpha_{\xi}<\alpha<\lambda$ for each $\xi$.

Lemma 3. If $E$ is a linear set such that $\bar{E} \equiv \lambda$, then $E$ contains a Borel set of power $2^{N_{0}}$.

(10) See $\$ 4$. 
Proof. Let $f$ be a similarity transformation of $R$ into $E$. Since $f$ is a similarity transformation, it is a Baire function. If $g$ is a one to one Baire function defined on a Borel set, then $g(G)$ is a Borel set [7, p. 397]. Therefore $f(R)$ is a Borel set of power $2^{\aleph_{0}}$.

Lemma 4. There exists a family $G=\left\{B_{\xi} \mid \xi<\theta\right\}$ of pairwise disjoint linear sets such that $\bar{B}_{\xi} \equiv \lambda$ for each $\xi$. Furthermore, $G$ has the property that for each nondenumerable Borel set $B$, an ordinal number $v<\theta$ can be found so that $B \cap B_{v}$ contains a nondenumerable Borel set.

Proof. If $B$ is a nondenumerable Borel set, then $\bar{B} \equiv \lambda$. This is so since each nondenumerable Borel set contains a nonempty perfect set $[7$, p. 355]. Each nondenumerable Borel set is thus of power $2^{\aleph_{0}}$. Let $H=\left\{P_{\xi} \mid \xi<\theta\right\}$ be a family of pairwise disjoint, nondenumerable, perfect sets. Being a perfect set, each $P_{\xi}$ is a Borel set. Let the family of those nondenumerable Borel sets, which are not elements of $H$, be well ordered into the sequence

$$
Q_{0}, Q_{1}, \cdots, Q_{\xi}
$$

Denote by $R_{0}$ the first element in (1) which is almost disjoint with each element of $H\left({ }^{11}\right)$. Suppose that for each $v<\gamma$, where $\gamma<\theta$, an element $R_{v}$ of (1) has been defined which is almost disjoint with each element in the family $H \cup\left\{R_{\xi} \mid \xi<v\right\}$. Let $R_{\gamma}$ be the first element in (1), if one exists, which is almost disjoint with each element of the family $H \cup\left\{R_{\xi} \mid \xi<\gamma\right\}$. By transfinite induction this procedure is continued until either $R_{\xi}$ is defined for each $\xi<\theta$, or else, for some ordinal number $\gamma$, the set $R_{\gamma}$ does not exist.

Consider the family of sets $\left\{S_{\xi} \mid \xi<\theta\right\}=H \cup\left\{R_{\xi} \mid \xi<\gamma \leqq \theta\right\}$. From the selection of the sets $R_{\xi}$, it follows that for each nondenumerable Borel set $B$, there exists an ordinal number $v$ such that $B \cap S_{v}$ is nondenumerable. As the intersection of two Borel sets is a Borel set, $B \cap S_{v}$ is a nondenumerable Borel set. Denote by $B_{0}$, the set $S_{0}$, and for each $v<\theta$, by $B_{v}$ the set

$$
S_{v}-\left[\bigcup_{\xi<v}\left(S_{c} \cap S_{\xi}\right)\right] \text {. }
$$

Note that the $B_{v}$ are pairwise disjoint. If $S_{\xi} \cap S_{v}$ is nondenumerable, being a Borel set it is of power $2 \aleph_{0}$. As the $S_{\xi}$ are almost disjoint, it follows that $S_{\xi} \cap S_{v}$ is denumerable. Combining this with the fact that $v<\theta$, we see that the set $\bigcup_{\xi<v}\left(S_{\xi} \cap S_{v}\right)$ is of power $<2^{\aleph_{0}}$. In [11] Sierpiński has shown that if $\bar{P} \equiv \lambda$, then on removing $<2^{N_{0}}$ elements from $P$, the resulting set is $\equiv \lambda$. Consequently $\bar{B}_{v} \equiv \lambda$. Now let $B$ be any nondenumerable Borel set. For some ordinal number $v, B \cap S_{v}$ is a nondenumerable Borel set, thus $\overline{B \cap}_{v} \equiv \lambda$. Since the power of $S_{v}-B_{v}$ is $\left\langle 2 \aleph^{\aleph_{0}}, \overline{B \cap}_{v} \equiv \lambda\right.$. From Lemma 3, $B \cap B_{v}$ contains a Borel set of power $2^{\aleph_{0}}$.

(11) A set $A$, of power $2^{\aleph_{0}}$, is almost disjoint with a family of sets $Q$, each element of $Q$ being of power $2 \aleph_{0}$, if, for each element $P$ in $Q$, the power of $A \cap P$ if $<2 \aleph_{0}$. 
REMARK. If $20=\boldsymbol{\aleph}_{1}$, then the sets $B_{\xi}$ obtained above are Borel sets.

Lemma 5. Let $\left\{\alpha_{\xi}\right\}, \xi<\theta$, be a sequence of order types, each $\alpha_{\xi}$ being $<\lambda$. There exists a linear set $E$ with the following two properties: (1) $\bar{E}<\lambda$; and (2) $E$ is the union of a family $\left\{E_{\xi} \mid \xi<\theta\right\}$ of pairwise disjoint sets, where $\bar{E}_{\xi}=\alpha_{\xi}$ for each $\xi$.

Proof. Let $G=\left\{B_{\xi} \mid \xi<\theta\right\}$ be a family of pairwise disjoint sets, where $\bar{B}_{\xi} \equiv \lambda$ for each $\xi$. Let $G$ have the property that for each nondenumerable Borel set $B$ an ordinal number $v<\theta$ can be found so that $B \cap B_{v}$ contains a nondenumerable Borel set. The existence of such a family is guaranteed by Lemma 4. For each $\xi$ let $E_{\xi}$ be a subset of $B_{\xi}$ such that $\bar{B}_{\xi}=\alpha \xi$, and let $E=U_{\xi<\theta} E_{\xi}$. In order to see that $\bar{E}<\lambda$, suppose the contrary, i.e., suppose that $\bar{E} \equiv \lambda$. Let $f$ be a similarity transformation of $R$ into $E$. Since $f(R)$ is a Borel set of power $2 \aleph_{0}$, for some ordinal number $v, f(R) \cap B_{v}$ contains a nondenumerable Borel set $A$. Since $\bar{A} \equiv \lambda, \overline{f(R) \cap B_{v}} \equiv \lambda$. As the $B_{\xi}$ are pairwise disjoint sets and $f(R)$ is a subset of $E$,

$$
\lambda \equiv \overline{f(R) \cap B_{v}} \leqq \overline{E \cap B_{v}}=\bar{E}_{v} .
$$

This is a contradiction since $\bar{E}_{v}=\alpha_{v}<\lambda$. Consequently no such function $f$ can exist, i.e., $\bar{E}<\lambda$. Q.E.D.

In view of the fact that for any order type $\alpha$ which is $<\lambda$ there exists an exact order type $\tau$ such that $\alpha<\tau<\lambda[3]$, we have the following result:

Theorem 15. For any sequence $\left\{\alpha_{\xi}\right\}, \xi<\theta$, of order types, each $\alpha_{\xi}$ being $<\lambda$, there exists an exact order type $\tau$ such that $\alpha_{\xi}<\tau<\lambda$ for each $\xi$.

Corollary. Let $\alpha$ be any order type which is $<\lambda$. Problem $\mathrm{P}$, as applied to $\sigma=\alpha$ and $\mu=\lambda$, admits of a well ordered set, of power $>2 \aleph^{\aleph_{0}}$, of exact solutions, each solution being of power $2^{\aleph_{0}}$.

Turning briefly to denumerable order types we have

THEOREM 16. Let $\left\{\alpha_{n}\right\}, n<\omega$, be a sequence of denumerable order types, where each $\alpha_{n}$ is $<\eta$. Then $\alpha<\eta$, where $\alpha=\alpha_{0}+\alpha_{1}+\cdots+\alpha_{n}+\cdots$.

Proof. Denote by $S$ the set of rational numbers, ordered in the natural manner. For each integer $n \geqq 0$, let $A_{n}$ be a subset of $S \cap(n, n+1)$, such that $\bar{A}_{n}=\alpha_{n}$. Now in [8] it was shown that if $E$ is a denumerable set, then a necessary and sufficient condition that $\bar{E}$ be $\equiv \eta$ is that for each denumerable ordinal number $\beta, E$ contain a set $D_{\beta}$ such that $\bar{D}_{\beta}=\beta$. For each $n$ let $\beta_{n}$ be the smallest ordinal number which has the property that $A_{n}$ contains no subset of ordinal type $\beta_{n}$. Since $\alpha_{n}$ is assumed $<\eta, \beta_{n}<\omega_{1}$. Let $\beta=\beta_{0}+\beta_{1}$ $+\cdots+\beta_{n}+\cdots$. Being the sum of a denumerable number of denumerable ordinal numbers, $\beta$ is $<\omega_{1}$. Consider the order type $\beta+1$. The set $F=\bigcup_{n<\omega} A_{n}$ does not contain a set of order type $\beta+1$ [8]. Therefore $\alpha=\bar{F}<\eta$. Q.E.D. 
In [3] it was shown that if $\alpha<\eta$ then there exists an order type $\tau$ such that $\alpha<\tau<\eta$. From this we conclude

Corollary. Let $\alpha$ be any denumerable order type which is $\langle\eta$. Problem P, as applied to $\sigma=\alpha$ and $\mu=\eta$, admits of a set, of order type $\omega_{1}$, of solutions.

The following problem has been open for several years.

(1) For $\sigma=0$ and $\mu=\eta$, does problem $\mathrm{P}$ admit of a set of solutions of order type $\omega^{*}$ ?

Two open questions related to (1) are

(2) Does there exist a denumerable set which has $\boldsymbol{\aleph}_{0}$ fixed points?

(3) Do there exist $\boldsymbol{\aleph}_{0}$ incomparable denumerable order types? A positive solution to (2) would imply a positive solution to both (1) and (3).

\section{Schisms.}

Definition. Let $A$ and $B$ be two simply ordered sets such that $\bar{A}<\bar{B}$. If $A$ is the ordered sum $A=F+G$, such that $\bar{F}+1+\bar{G} \| \bar{B}$, then the ordered pair $(\bar{F}, \bar{G})$ is called a schism of $(\bar{A}, \bar{B})$.

For $\bar{A}<\bar{B}$ it is not always true that $(\bar{A}, \bar{B})$ has a schism. One need only examine the case where $\bar{A}<\bar{A} 2=\bar{B}$. To consider a situation where a schism occurs, let an element $p$ in $A$ be called a fixed $(A, B)$ point if there exists an element $q$ in $B$ such that $f(p)=q$ for every similarity transformation $f$ of $A$ into $B$. Then $(\bar{A}, \bar{B})$ has a schism if there exists a fixed $(A, B)$ point.

The assumption of the existence of a fixed $(A, B)$ point $p$ is a strong one, implying in particular that $p$ is a fixed point of $A$. As to a sufficiency condition for a fixed $(A, B)$ point the following results are easily shown:

(1) Let $\bar{A} \omega<\bar{B} \omega$. Let the two relations, $(\alpha) \bar{A} \omega<\bar{B} n$ and $(\beta) \bar{A} \omega \equiv \bar{B} n$ both be false for every positive integer $n$. If $\bar{E}=\bar{A} \omega+1$ and $\bar{F}=\bar{B} \omega+1$, then $p$ is a fixed $(E, F)$ point, where $p$ is the last element of $E$.

(2) Let $E$ have properties $A$ and $C$. If $B$ is a subset of $E$ such that, for any two elements $a<b$ in $B$, the power of the set $\{x \mid a<x<b, x \in B\}$ is $2 \aleph_{0}$, then each element of $B$ is a fixed $(B, E)$ point.

We now show that the concept of a schism is invariant under " $\equiv$ " of order types.

THEOREM 17. Let $\left(\alpha^{\prime}, \beta^{\prime}\right)$ be a schism of $\left(\gamma^{\prime}, \delta^{\prime}\right)$. Then for each pair of order types $\gamma \equiv \gamma^{\prime}$ and $\delta \equiv \delta^{\prime}$, there exists a pair of order types $\alpha$ and $\beta$ such that $\alpha \equiv \alpha^{\prime}$, $\beta \equiv \beta^{\prime}$, and $(\alpha, \beta)$ is a schism of $(\gamma, \delta)$.

Proof. Let $E^{\prime}$ be a simply ordered set which contains two subsets $C^{\prime}$ and $D^{\prime}$ whose order types are $\gamma^{\prime}$ and $\delta^{\prime}$ respectively. As $\left(\alpha^{\prime}, \beta^{\prime}\right)$ is a schism of $\left(\gamma^{\prime}, \delta^{\prime}\right)$, it follows that $C^{\prime}$ is the ordered sum of two subsets $A^{\prime}$ and $B^{\prime}$, whose order types are $\alpha^{\prime}$ and $\beta^{\prime}$ respectively. Denote by $p$ some abstract element which is not in $E^{\prime}$. Let $p$ follow each element in $A^{\prime}$ and precede each element of $B^{\prime}$. Thus the order type of the set $A^{\prime} \cup\{p\} \cup B^{\prime}$ is $\alpha^{\prime}+1+\beta^{\prime}$. Now the two facts, (1) $\alpha^{\prime}+\beta^{\prime}<\delta^{\prime}$ and (2) $\alpha^{\prime}+1+\beta^{\prime}$ and $\delta^{\prime}$ are incomparable, together imply that $\alpha^{\prime}+\beta^{\prime}<\alpha^{\prime}+1+\beta^{\prime}$. Therefore by Theorem 2.1 of [3] $p$ is a fixed 
point of the set $A^{\prime} \cup\{p\} \cup B^{\prime}$. Let $D$ be any set whose order type is $\delta$ and $C$ a subset of $D$ whose order type is $\gamma$. As $\bar{C} \equiv \bar{C}^{\prime}$, there exists a similarity transformation $f$ of $C$ into $C^{\prime}$, and a similarity transformation $g$ of $C^{\prime}$ into $C$. Let $A=\left\{x \mid f(x) \in A^{\prime}\right\}$ and $B=\left\{x \mid f(x) \in B^{\prime}\right\}$. Clearly $C$ is the ordered sum $A+B$. We shall now show that $\bar{A} \equiv \bar{A}^{\prime}$ and $\bar{B} \equiv \bar{B}^{\prime}$. This will imply that $(\bar{A}, \bar{B})$ is a schism of $(\bar{C}, \bar{D})$.

To accomplish this it will be sufficient to show that (a) $g\left(A^{\prime}\right)$ is a subset of $A$ and (b) $g\left(B^{\prime}\right)$ is a subset of $B$. Therefore suppose that either (a) or (b) is false, say (a). Hence the set $g\left(A^{\prime}\right) \cap B$ is nonempty. Let $r$ be some element of $g\left(A^{\prime}\right) \cap B$. Denote by $h$ the function which is defined by $h(x)=g(x)$ for $x$ in $A^{\prime}, h(p)=g f(r)$, and $h(x)=g f g(x)$ for $x$ in $B^{\prime}$. Then $h$ is a similarity transformation of $A^{\prime} \cup\{p\} \cup B^{\prime}$ into $C$. This contradicts the assumption that $\bar{C}<\bar{A}^{\prime}+1+\bar{B}^{\prime}$. An analogous contradiction arises upon assuming that condition (b) is false. Therefore (a) and (b) both must be true. Q.E.D.

In preparation for Theorem 18 the following result is needed.

Lemma 6. Let $A$ be a proper subset of $B$ and $p$ a fixed point of $A$. Then $p$ is a fixed point of the set $C=A \cup\{q\}$ for each element $q$ of $B-A$.

Proof. Suppose that $p$ is not a fixed point of $C$, i.e., suppose there exists a similarity transformation $g$ of $C$ into $C$ for which $g(p) \neq p$. We shall show that this implies the existence of a similarity transformation $f$ of $A$ into $A$ for which $f(p) \neq p$, thus obtaining a contradiction. We shall suppose that $q<p$, an analogous treatment being possible if $q>p$. Several possibilities arise:

(a) For no element $y$ in $C$ does $g(y)=q$. Let $f(x)=g(x)$ for each element $x$ in $A$.

(b) There exists an element $y$ in $C$ such that $g(y)=q$.

(1) If $y>q$, let $f(x)=g(x)$ for $x>y$, and $f(x)=g[g(x)]$ for those elements $x$ in $A$ which are $\leqq y$.

(2) If $y=q$, let $f(x)=g(x)$ for each element $x$ in $A$.

(3) If $y<q$, let $f(x)=x$ for $x \leqq y$, and $f(x)=g(x)$ for those elements $x$ in $A$ which are $>y$.

In each case the function $f$ is a similarity transformation of $A$ into $A$ for which $f(p) \neq p$.

TheOREM 18. Let $A$ be a simply ordered set containing two fixed points $p$ and $q$ which have the property that the power of the set $\{x \mid p<x<q, x \in A\}$ is infinite. Then there exists a schism of $(\overline{A-\{p\}}, \bar{A})$, and a schism of $(\overline{A-\{q\}}$, $\bar{A})$.

Proof. It will be sufficient to exhibit a schism of $(\overline{A-\{p\}}, \bar{A})$, a similar exhibition being possible for $(\overline{A-\{q\}}, \bar{A})$.

Let $F=\{x \mid x<q, x \in A-\{p\}\}$ and $G=\{x \mid x \geqq q, x \in A\}$. For some abstract element $r$ which is not in $A$, let $r$ follow each element in $F$ and precede each element in $G$. Then $(\bar{F}, \bar{G})$ is a schism of $(\overline{A-\{p\}}, \bar{A})$, i.e., $\overline{F \cup\{r\} \cup G} \| \bar{A}$. 
To see this let $B=F \cup\{r\} \cup G$. Suppose that there exists a similarity transformation $f$ of $A$ into $B$. If $f(p)<p$, let $g(x)=f(x)$ for $x \leqq p$, and $g(x)=x$ for $x>p$. Suppose that $f(p)>p$. Several possibilities arise:

(1) There exists an element $s$ such that $p \leqq s<r$ and $f(s)>r$. Let $g(x)$ $=f(x)$ for $x<r, g(r)=f[f(s)]$, and $g(x)=f[f(x)]$ for $x>r$.

(2) $p<f(x)<r$ for each element $x$ satisfying $p \leqq x<r$. Let $g(x)=f(x)$ for each element $x<r$ and $g(x)=x$ for $x \geqq r$.

(3) $p<f(x) \leqq r$ for each element $x$ satisfying $p \leqq x<r$, and for some element $x_{0}, f\left(x_{0}\right)=r$. It follows that $x_{0}$ and $r$ are consecutive elements in the set $A \cup B$, i.e., there is no element $x$ in $A \cup B$ such that $x_{0}<x<r$. By Lemma 6 then, $q$ is a fixed point of $A \cup B$. Thus $r$ is a fixed point of $A \cup B$. Thus there is a maximal set of consecutive elements of $A \cup B$ in which $r$ is the last element. Clearly this set must be finite. Let the elements of this maximal set be $x_{n}<x_{n-1}<\cdots<x_{0}<r$. Since the power of the set $\{x \mid p<x<q, x \in A\}$ is infinite, there are an infinite number of elements $x$ for which $p<x<x_{n}$. Since there is no immediate predecessor of $x_{n}$ in $A \cup B$, if there were an element $t$ such that $p<t<x_{n}$ and $f(t) \geqq x_{n}$, then an infinite set of elements, namely the set $\{x \mid t \leqq x \leqq r\}$, would be mapped by $f$ into the finite set of elements $\left\{x_{n}, x_{n-1}, \cdots, x_{0}, r\right\}$. As this violates $f$ being a one to one function, it follows that $p<f(x)<x_{n}$ for $p \leqq x<x_{n}$. Let $g(x)=f(x)$ for $x<x_{n}$ and $g(x)=x$ for $x \geqq x_{n}$.

In each of the above cases we have constructed a similarity transformation $g$ of $A \cup B$ into $A \cup B$ such that $g(p) \neq p$. By Lemma 6 however, $p$ is a fixed point of $A \cup B$. From this contradiction we see that there can be no similarity transformation $f$ of $A$ into $B$.

In a similar manner, by investigating $f(r)$, it can be shown that there is no similarity transformation $f$ of $B$ into $A$. This, combined with the fact that there is no similarity transformation of $A$ into $B$, proves that $\bar{A} \| \bar{B}$. Thus $(\bar{F}, \bar{G})$ is a schism of $(\overline{A-\{p\}}, \bar{A})$.

REMARK. If the assumption of the existence of a second fixed point $q$ in Theorem 18 is removed, then the conclusion may be false. This is so, for example, in a simply ordered set of order type $1+\omega^{*}$. Likewise the conclusion is false if the assumption that there are an infinite number of elements between the two fixed points is removed. This is so in a set of order type $\omega+2$.

Corollary. Let $A$ be an infinite, exact set, and let $p$ be any element of $A$. Then there exists a schism of $(\overline{A-\{p\}}, \bar{A})$.

5. Consequences of Theorem 18. The demonstration given for Theorem 18 essentially has proved the following result.

Theorem 19. Let $p$ and $q, p<q$, be two fixed points of the set $A$. If the set $\{x \mid p<x<q, x \in A\}$ is infinite, then $\overline{A-\{p\}} \| \overline{A-\{q\}}$.

From Theorem 19 and the fact that, by Corollary 2 of Theorem 2, if $A$ 
is exact and also a subset of $B$, then $A \cup\{p\}$ is exact for each element $p$ in $B$, there follows

Theorem 20. Let $A$ be a subset of $B$ and $p$ and $q$ any two elements of $B-A$. If $A$ is exact and if the power of the set $\{x \mid p<x<q, x \in A\}$ is infinite, then $\overline{A \cup\{p\}} \| \overline{A \cup\{q\}}$.

CoRollary 1. Let $A$ be an exact subset of $R$, and $p$ and $q$ any two elements of $R-A$ for which the power of the set $\{x \mid p<x<q, x \in A\}$ is infinite. Then $\overline{A \cup\{p\}} \| \overline{A \cup\{q\}}$.

In Theorem 4 it was shown that each linear set of power $2^{\aleph_{0}}$ contains an exact subset which has property $\mathrm{C}$. Hence

COROLlary 2. Each linear set of power $2^{\mathrm{N}_{0}}$ contains a family of subsets $\left\{E_{\xi} \mid \xi<\theta\right\}$ such that each set is exact and of power $2 \mathrm{~N}_{0}$, the order types of the sets are pairwise incomparable, and the symmetric difference of the sets is two elements.

The following result is a generalization of Theorem 5 in [11].

Corollary 3. Let $A$ be a dense, exact, subset of $R$. If $p$ and $q$ are any two distinct elements of $R-A$, then $\overline{A \cup\{p\}} \| \overline{A \cup\{q\}}$.

REMARK. In reply to a question of the referee, the power of $R-A$ is $2^{N_{0}}$. This result is due to Sierpiński [11] since $\bar{A}<\lambda$. Thus there are $2^{\aleph_{0}}$ such points $p$ and $q$.

In [3] it was shown that $R$ is the union of two disjoint, exact, dense (in $R$ ), subsets $G$ and $H$, of power $2{ }^{N_{0}}$ each. From this we get

Corollary 4. There exist two disjoint linear sets $G$ and $H$, of power $2^{\mathrm{N}_{0}}$ each, with the following properties:

(1) $G \cup H=R$;

(2) for any two elements $p$ and $q$ in $H, \overline{G \cup\{p\}} \| \overline{G \cup\{q\}}$;

(3) for any two elements $p$ and $q$ in $G, \overline{H \cup\{p\}} \| \overline{H \cup\{q\}}$.

Corollary 5. To each set of order types $\left\{\alpha_{\xi} \mid \xi<\gamma<\theta\right\}$, where $\alpha_{\xi}<\lambda$ for each $\xi$, there corresponds a family of exact sets $\left\{A_{\xi} \mid \xi<\theta\right\}$, a set of points $\left\{p_{\xi} \mid \xi<\theta\right\}$, and $a$ set $A$ with the following three properties: (1) $\alpha_{\xi}<\bar{A}_{\xi}<\lambda$; (2) $A_{\xi}=A \cup\left\{p_{n}\right\}$; and (3) the order types of the $A_{\xi}$ are pairwise incomparable.

Corollary 5 follows from 2.4 of [3] and Theorem 15 .

One method for obtaining the points $p$ and $q$ which are described in Theorem 19 is to apply the next lemma.

Lemma 7. Let $\bar{A} \| \bar{B}$. If $p$ is a fixed point in the ordered sum $\{p\}+A$, and $q$ is a fixed point in the ordered sum $B+\{q\}$, then $p$ and $q$ are both fixed points in the ordered sum $D=\{p\}+A+B+\{q\}$. Furthermore, the set $F=\{x \mid p<x<q$, $x \in D\}$ is infinite. 
Proof. Since the order types of any two simply ordered sets, one of which is finite, are comparable, it follows that the sets $A$ and $B$ are both infinite. Thus the set $F$ is infinite. Suppose that one of the points, say $p$, is not a fixed point of $D$. Hence there exists a similarity transformation of $D$ into $D$ such that $f(p)>p$. Denote by $G$ the set

$$
G=\bigcup_{n<\omega}\left\{x \mid f^{n}(p) \leqq x<f^{n+1}(p), x \in D\right\} .
$$

Suppose that $G$ is a subset of $A$. Then the function $g$, which is defined by $g(x)=f(x)$ for $x$ in $G$ and $g(x)=x$ for $x$ in $A-G$, is a similarity transformation of $\{p\} \cup A$ into $\{p\} \cup A$ such that $f(p) \neq p$. From this contradiction of $p$ not being a fixed point we see that there exists a point $y$ in $G \cap B$. This implies that for some positive integer $n, f^{n}(p)$ is in $B$. Consider the similarity transformation $f^{n}$. It maps $A$ into $B$. This contradicts the fact that $\bar{A} \| \bar{B}$. Therefore $p$ must be a fixed point in $D$. An analogous argument shows that $q$ is a fixed point in D. Q.E.D.

REMARKs. (1) If the incomparability of the order types $\bar{A}$ and $\bar{B}$ is removed, then one can only conclude that one of the points, $p$ or $q$, must be fixed. This is a special case of the following result whose proof is in [5].

"If $p$ and $q$ are fixed points of $A$ and $B$ respectively, then either $p$ or $q$ is a fixed point in the ordered sum $A+B$."

(2) The converse to Lemma 7 is not true, i.e., the following is false:

(*) Let $p$ and $q, p<q$, be two fixed points of the simply ordered set $D$. If the set $F=\{x \mid p<x<q, x \in D\}$ is infinite, then $F$ is the ordered sum of two sets $A$ and $B$ whose order types are incomparable.

Let $E$ be an infinite exact subset of $(0,1)$ and $H$ a subset of $(1,2)$ which is similar to $E$. Let $D=\{0,2\} \cup E \cup H$. Now the set $D$ is exact so that 0 and 2 are both fixed points in $D$. Clearly $F$ is infinite. If $F$ is the ordered sum $A+B$ then either $A \subseteq E$ and $H \subseteq B$, or else $E \subseteq A$ and $B \subseteq H$. Thus $\bar{A} \| \bar{B}$. Consequently the conclusion in $\left(^{*}\right)$ is false.

We conclude this section with a simple consequence of Lemma 7 and Theorem 19. Let $\alpha$ and $\beta$ be two transfinite ordinal numbers. Then $1+\alpha^{*}+\beta$ $\| \alpha^{*}+\beta+1$.

6. On the converse of Theorem 19. The converse of Theorem 19 is also true as we shall now show. A generalization of the converse will then be given (Theorem 21).

Lemma 8. If $\bar{B} \| \bar{C}$, and if the symmetric difference, $(B-C) \cup(C-B)$, contains just two elements, $p$ and $q$, where $p<q$ in the set $D=B \cup C$, then

(1) $p$ and $q$ are both fixed points in $D$;

(2) there exists a set $A$ such that $B=A \cup\{y\}$ and $C=A \cup\{z\}$, where $y$ is one of the points $p$ or $q$ and $z$ is the other point; and

(3) the set $F=\{x \mid p<x<q, x \in D\}$ is infinite.

Proof. Let $A=B-\{y\}$ where $y$ is the element $p$ or $q$ which is in $B$. (2) is 
now obvious. As to (1), if $y$ is not a fixed point of $D=A \cup\{y\} \cup\{z\}$, then by Theorem 2.1 of $[3], \bar{D} \equiv \overline{A \cup\{z\}}=\bar{C}$. Thus $\bar{B} \leqq \bar{D} \equiv \bar{C}$, a contradiction. Similarly $z$ is a fixed point of $D$. As to (3), suppose that $F$ is finite, its elements being

$$
p=x_{0}<x_{1}<\cdots<x_{n}=q .
$$

Let $f$ be the function which is defined as follows: $f(x)=x$ if $x<p$ or $x>q$, and $f\left(x_{i}\right)=x_{i+1}$ for $i<n$. Then $f$ is a similarity transformation of one of the sets $B$ or $C$ onto the other, i.e., $\bar{B}=\bar{C}$. This contradicts the assumption that $\bar{B} \| \bar{C}$. Therefore $F$ must be infinite.

THEOREM 21. Let $H=\left\{A_{i} \mid i \leqq n\right\}$ be a family of $n+1$ sets with the following two properties: $(\alpha)$ The order types of the $A_{i}$ are pairwise incomparable; and $(\beta)$ the symmetric difference of each pair of sets $A_{i}$ and $A_{j}, i \neq j$, contains just two elements. Then one of two possibilities occurs.

(A) There exists a unique set of $n+1$ elements, $\left\{p_{i} \mid i \leqq n\right\}$, and a unique set $A$, such that

(1) $p_{i}$ is a fixed point in the set $D=\bigcup_{i \leqq n} A_{i}$;

(2) $A_{i}=A \cup\left\{p_{i} \mid i \leqq n\right\}-\left\{p_{i}\right\}=D-\left\{p_{i}\right\}$; and

(3) if $p_{i}<p_{j}$ then the set $F_{i j}=\left\{x \mid p_{i}<x<p_{j}, x \in D\right\}$ is infinite.

(B) There exists a unique set of $n+1$ elements $\left\{t_{i} \mid i \leqq n\right\}$, and a unique set $A$, such that

(1) $t_{i}$ is a fixed point of the set $D=\cup_{i \leq n} A_{i}$;

(2) $A_{i}=A \cup\left\{t_{i}\right\}=\left[D-\left\{t_{j} \mid j \leqq n\right\}\right] \cup\left\{t_{i}\right\}$; and

(3) if $t_{i}<t_{j}$ then the set $F_{i j}=\left\{x \mid t_{i}<x<t_{j}, x \in D\right\}$ is infinite.

Furthermore, if $n \geqq 3$, then every subfamily of $H$, consisting of three or more sets, satisfies the same set of conclusions, (A) or (B), as does the family $H$.

Proof. It is evident, in view of Lemma 8, that in both (A) and (B), (1), (3), and the uniqueness of the sets will follow from (2). We shall now consider (2). For any two sets $E$ and $F$ denote the symmetric difference of the sets by $E \Delta F$. Notice that for $i \neq j, A_{i}-A_{j}$ is a set consisting of just one element.

By Lemma 8 the theorem is true for $n=1$. Continuing by mathematical induction suppose that the theorem is true for $n=k$. We shall demonstrate our result if we show that the theorem is true for $n=k+1$.

By the induction hypothesis either (A) or (B) holds for $n=k$.

First suppose that (A) holds. Thus there exists a set $B$ and a set of $k+1$ points, $\left\{p_{i} \mid i \leqq k\right\}$, such that $A_{i}=B \cup\left\{p_{i} \mid i \leqq k\right\}-\left\{p_{i}\right\}$ for $i \leqq k$. Denote by $q$ the element for which $A_{0}-A_{k+1}=\{q\}$. Two alternatives arise:

(a) Suppose that $q \neq p_{i}$ for $i \leqq k$. Denote by $p_{k+1}$ the element $q$. Since $p_{k+1}$ is in $A_{0}-A_{k+1}$ and $p_{k+1} \neq p_{i}$ for $i \leqq k$, it follows that $p_{k+1}$ is in $B$. Signifying by $A$ the set $B-\left\{p_{k+1}\right\}$, we have

$$
A_{i}=A \cup\left\{p_{j} \mid j \leqq k+1\right\}-\left\{p_{i}\right\} \quad \text { for } i \leqq k .
$$


Let $\{r\}=A_{k+1}-A_{0}$. Suppose that $r \neq p_{0}$. Then $r$ is not in $A_{0} \cup\left\{p_{0}\right\}$, thus not in $A_{1}$ (since $k \geqq 1$ ). Hence $\{r\}=A_{k+1}-A_{1}$. Combining this with the fact that $\left\{p_{k+1}\right\}=A_{1}-A_{k+1}$ we get $A_{k+1} \Delta A_{0}=A_{k+1} \Delta A_{1}$. Now it is well known that if $E \Delta F=E \Delta G$, then $F=G$. Therefore $A_{0}=A_{1}$, a contradiction. Consequently $r=p_{0}$. Thus

$$
A_{k+1} \subseteq A_{0} \cup\left\{p_{0}\right\}=A \cup\left\{p_{i} \mid i \leqq k+1\right\}
$$

Since $p_{k+1}$ is not in $A_{k+1}$ it follows that $A_{k+1} \subseteq A \cup\left\{p_{i} \mid i \leqq k\right\}$. As $\left\{p_{k+1}\right\}$ $=A_{0}-A_{k+1}$, we get $A_{0} \subseteq A_{k+1} \cup\left\{p_{k+1}\right\}$, or what is the same, $A_{0}-\left\{p_{k+1}\right\}$ $\subseteq A_{k+1}$. This, together with the fact that $p_{0}$ is in $A_{k+1}$, implies that $A \cup\left\{p_{i} \mid i\right.$ $\leqq k\} \subseteq A_{k+1}$. Thus $A_{k+1}=A \cup\left\{p_{i} \mid i \leqq k\right\}$.

(b) Suppose that $q=p_{i}$ for some integer $i \leqq k$. As $p_{0}$ is not in $A_{0}$, it follows that $0<i \leqq k$. Thus

$$
\left\{p_{i}\right\}=A_{0}-A_{i}=A_{0}-A_{k+1} .
$$

Suppose that $p_{0}$ is in $A_{k+1}$. Then

$$
\left\{p_{0}\right\}=A_{k+1}-A_{0}=A_{i}-A_{0} .
$$

Hence $A_{0} \Delta A_{k+1}=A_{0} \Delta A_{i}$. Thus $A_{i}=A_{k+1}$, a contradiction. Therefore $p_{0}$ is not in $A_{k+1}$. Let $\{r\}=A_{k+1}-A_{0}$. Since $r$ is in $A_{k+1}$, it follows that $r \neq p_{0}$ and $r \neq p_{i}$. Suppose that $k>1$. Let $j$ be an integer $\leqq k$ which is distinct from 0 and $i$. Then the set $A_{j}-A_{k+1}$ contains the two elements $p_{0}$ and $p_{i}$. This contradicts the fact that $A_{j}-A_{k+1}$ contains just one element. Consequently, if $k>1$, then only case (a) above can exist. Suppose that $k=1$. Then $i=1$ and $A_{k+1}=A_{2}$. From $\{r\}=A_{2}-A_{0}$ we get $A_{2} \subseteq A_{0} \cup\{r\}$. Since $p_{1}$ is not in $A_{2}$, it follows that $A_{2} \subseteq B \cup\{r\}$. From $\left\{p_{1}\right\}=A_{0}-A_{2}$ we get $A_{0} \subseteq A_{2} \cup\left\{p_{1}\right\}$, i.e., $B \subseteq A_{2}$. As $r$ is in $A_{2} ; B \cup\{r\} \subseteq A_{2}$. Hence $A_{2}=B \cup\{r\}$. Denoting by $A$ the set $B$ and by $t_{0}, t_{1}$, and $t_{2}$, the elements $p_{1}, p_{0}$, and $r$ respectively, we obtain (B).

Now suppose that (B) holds. Since the case where $k=1$ has been handled under (A) we shall assume that $k \geqq 2$. Thus, there exists a set of $k+1$ elements, $\left\{t_{i} \mid i \leqq k\right\}$, and a set $A$, such that $A_{i}=A \cup\left\{t_{i}\right\}$ for $i \leqq k$. Let $\{r\}=A_{k+1}-A_{0}$.

(c) Suppose that $r \neq t_{i}$ for $i \leqq k$. Therefore $r$ is not in $A_{i}$ for $i \leqq k$. Thus $A_{k+1}-A_{i}=\{r\}$, so that $A_{k+1} \subseteq A_{i} \cup\{r\}$. From this we get $A_{k+1} \subseteq A \cup\{r\}$. This, together with $t_{i}$ not being $r$, yields $A_{i}-A_{k+1}=\left\{t_{i}\right\}$. Thus $A_{i} \subseteq A_{k+1}$ $\cup\left\{t_{i}\right\}$, i.e., $A \subseteq A_{k+1}$. Since $r$ is in $A_{k+1}, A \cup\{r\} \subseteq A_{k+1}$. Consequently $A \cup\{r\}=A_{k+1}$. Denote by $t_{k+1}$ the element $r$. In this case then (B) is satisfied.

(d) Suppose that $r=t_{i}$ for some integer $i \leqq k$. As $t_{0}$ is in $A_{0}, i>0$. Thus

$$
A_{k+1} \subseteq A_{0} \cup\left\{t_{i}\right\}=A \cup\left\{t_{0}, t_{i}\right\} .
$$

Consider the element $q$, where $\{q\}=A_{0}-A_{k_{1} 1}$. Since $t_{i}$ is in $A_{k+1}, q \neq t_{i}$. Two possibilities arise.

First suppose that $q=t_{0}$. Then $A_{k+1} \subseteq A \cup\left\{t_{i}\right\}$. Let $\{s\}=A_{i}-A_{k+1}$. As $t_{i}$ is in $A_{k+1}, s$ must be in $A$. The set $A_{0}-A_{k+1}$ now contains the two elements $s$ and $t_{0}$. This is a contradiction. 
Now suppose that $q \neq t_{0}$, i.e., $t_{0}$ is in $A_{k+1}$. Since $k \geqq 2$ there exists an integer $j \leqq k$ which is distinct from 0 and 1 . The set $A_{k+1}-A_{j}$ contains the two elements $t_{0}$ and $t_{i}$. Again we have a contradiction.

Since each of the two possibilities leads to a contradiction, case (d) cannot occur, i.e., only case (c) can exist.

The remaining part of the conclusion follows from the fact that for $k \geqq 2$, the process of extending (A) or (B) to $k+1$ sets from $k$ sets always yields the same selection (A) or (B) for the $k+1$ sets as for the $k$ sets. Q.E.D.

REMARKS. (1) The generalization of Theorem 21 to the case in which $n$ is any ordinal number offers no difficulty. One merely replaces finite induction by transfinite induction.

(2) By virtue of Theorems 19 and 20 there exist families of sets where (A) holds and families of sets where (B) holds.

(3) The demonstration in Theorem 21 essentially proves the following result in general set theory: "Let $H=\left\{A_{\xi} \mid \xi<\alpha\right\}$ be a family of sets such that $A_{\xi}-A_{v}$ contains exactly one element for $\xi \neq v$. Then either (2) in (A) or (2) in (B) holds. Furthermore, if $\alpha \geqq 3$, then every subfamily of $H$, consisting of three or more sets, satisfies the same set of conclusions, (A) or (B), as does the family $H . "$

7. More on incomparable order types. Denote by $C$ the Cantor perfect set. Then $R-C$, being an open set, is the union of a family of pairwise disjoint open intervals $\left\{A_{n} \mid n<\omega\right\}$. Let $B$ be a dense subset of the open interval $(0,1)$ which has property $C$. Furthermore, let $B$ have property $A$. Now the set $B$ is evidently an ordered sum, $B=B_{0}+\cdots+B_{n}+\cdots$, where each set $B_{n}$ has the following properties:

(1) $B_{n}$ is a dense subset of some open interval $D_{n}$;

(2) $B_{n}$ has property $C$; and

(3) $B_{n}$ has property A.

For each $n$ let $E_{n}$ be a dense subset of $A_{n}$ which is similar to $B_{n}$. Denote by $G$ the set $G=\cup_{n<\omega} E_{n} . G$ is dense in $R-C$, thus dense in $R$. It is easy to see that $G$ has properties A and C. Now each $c$-condensation point in a set $M$ which has property $A$ is a fixed point of $M$, by Theorem 2.3 of [3]. Therefore $G$ is exact. Being perfect, $C$ contains a subset $H$ which is similar to $R$. From Corollary 2 of Theorem 20 there now follows

THEOREM 22. There exist two disjoint linear sets $G$ and $H$ with the following properties:

(1) $H$ is similar to the real numbers;

(2) $G$ is a dense, exact subset of $R$, which has properties $A$ and $C$;

(3) if $p$ and $q$ are any two elements of $H$, then $\overline{G \cup\{p\}} \| \overline{G \cup\{q\}}$.

Corollary. For each linear set $D$ there exists two disjoint sets $G$ and $H$ which have the two properties: (1) $\bar{H}=\bar{D}$, and (2) if $p$ and $q$ are any two elements of $H$, then $\overline{G \cup\{p\}} \| \overline{G \cup\{q\}}$. 
While discussing sets which have property A we mention

THEOREM 23. Each linear set $E$ of power $2^{\aleph_{0}}$ contains two disjoint sets $G$ and $H$, both having properties $\mathrm{A}$ and $\mathrm{C}$, and which have the following additional properties:

(1) $\bar{G} \| \bar{H}$

(2) for any two elements $p$ and $q$ in $H, \overline{G \cup\{p\}} \| \overline{G \cup\{q\}}$;

(3) for any two elements $p$ and $q$ in $G, \overline{H \cup\{p\}} \| \overline{H \cup\{q\}}$; and

(4) for any two elements $p$ in $G$ and $q$ in $H, \overline{G \cup\{q\}} \| \overline{H \cup\{p\}}$.

Proof. $E$ contains a set $D$ having properties $\mathrm{A}$ and C. As is well known $D$ can be decomposed into the union of two disjoint sets $G$ and $H$ which satisfy the two conditions: (1) $G$ and $H$ each have property C; and (2) $G$ and $H$ are each dense in $D$. There is no difficulty in verifying that the sets $G$ and $H$ satisfy the conclusions of Theorem 23.

REMARK. It is easily seen that instead of two sets $G$ and $H, m$ sets, where $2<m \leqq 2^{\aleph_{0}}$, could have been obtained, the sets satisfying the obvious generalizations of the conditions (1), (2), (3), and (4).

We conclude with some results on schisms.

THEOREM 24. Let $E$ be a linear set of power $2^{\aleph_{0}}$. Let $B$ be any subset of $E$, of power $2^{N_{0}}$, with the following two properties: (1) $\bar{B}<\bar{E}$; and (2) $E$ contains no two disjoint, similar sets, of power $2^{\aleph_{0}}$ each, whose order types are $\leqq \bar{B}$. Then there exists a schism of $(\bar{B}, \bar{E})$. Furthermore, a point $q$ in $R$ can be found so that $\overline{B \cup\{q\}} \| \bar{E}$.

Proof. Denote by $C$ the set of $c$-condensation points of $B$ which belong to $B$. Since $C$ has property $C$ and the set $E$ satisfies (2) above, it follows that the only similarity transformation of $C$ into $E$ is the identity transformation, i.e., each point of $C$ is a fixed $(C, E)$ point. Hence there exists a schism of $(\bar{C}, \bar{E})$. It remains to be shown that this schism can be affected by a point $q$ in $R$.

To see this let $D$ be a linearly dense, enumerable subset of $C$, i.e., if $x$ and $y$ are any two elements of $C$ for which $x<y$, then there exists an element $z$ in $D$ such that $x<z<y . D$ certainly exists since every dense in itself subset of $R$ contains such a set. Suppose that for each gap $(F, G)$ of $D\left({ }^{12}\right)$ there exists an element $x$ in $E$ such that $u<x<v$ for each element $u$ in $F$ and $v$ in $G$. Then, as is well known, $E$ contains a set which is similar to $R$, i.e., $\bar{E} \equiv \lambda$. Since $E$ satisfies condition (2) it is clear that $\bar{E} \equiv \lambda$ is false. Hence there must exist a gap $\left(F_{0}, G_{0}\right)$ of $D$ for which there is no element $x$ in $E$ such that $u<x<v$ for each element $u$ in $F_{0}$ and $v$ in $G_{0}$. Let $q$ be any element in $R-E$ for which $u<q<v$ for each element $u$ in $F_{0}$ and $v$ in $G_{0}$. Consider the set $B \cup\{q\}$. If $f$ is any similarity transformation of $B \cup\{q\}$ into $E$, then $f(x)=x$ for each

${ }^{(12)}(F, G)$ is a gap of $D$ if $D$ is the ordered sum of two nonempty sets $F$ and $G$, where $F$ has no last element and $G$ no first element. 
element $x$ in $C$. However the element $f(q)$ cannot be in $E$ since $u=f(u)<f(q)$ $<f(v)=v$ for each element $u$ in $F_{0}$ and $v$ in $G_{0}$. Thus no such transformation $f$ is possible. Since $\bar{B}<\bar{E}$ and since $\overline{B \cup\{q\}} \leqq \bar{E}$ is false, $\bar{B}<\overline{B \cup\{q\}}$. By Theorem 2.1 of [3], $q$ is a fixed point of $B \cup\{q\}$. Suppose that $\bar{E} \leqq \bar{B} \cup\{q\}$. Then $\bar{B}<\bar{E} \leqq \overline{B \cup\{q\}}$. By Theorem 2.2 of [3], there is no order type $\tau$ for which $\bar{B}<\tau<\overline{B \cup\{q\}}$. Thus $\bar{E} \equiv \overline{B \cup\{q\}}$, so that $\overline{B \cup\{q\}} \leqq \bar{E}$. From this contradiction we see that $\bar{E} \leqq \bar{B} \cup\{q\}$ is false. We conclude that $\overline{B \cup\{q\}} \| \bar{E}$. Q.E.D.

If $E$ has property A then condition (2) is satisfied for every subset $B$. Thus we get

Corollary. Let $E$ have property $\mathrm{A}$ and let $B$ be any subset such that $\bar{B}<\bar{E}$. Then there exists a schism of $(\bar{B}, \bar{E})$. Furthermore, a point $q$ in $R$ can be found so that $\overline{B \cup\{q\}} \| \bar{E}$.

REMARKs. (1) In general it is not true that if $E$ is a linear set and $B$ is a subset of $E$ for which a schism of $(\bar{B}, \bar{E})$ exists, then there must exist a point $q$ in $R$ such that $\overline{B \cup\{q\}} \| \bar{E}$. For example, it is not true in the case where

$$
\begin{gathered}
B=\{-1 / n \mid 1 \leqq n<\omega\} \cup\{0\} \cup\{1 / n \mid 1 \leqq n<\omega\} \\
\cup\{2-(1 / n) \mid 1 \leqq n<\omega\}
\end{gathered}
$$

and $E=B \cup\{2\}$.

(2) Theorem 24 may be modified to the extent of replacing, everywhere it occurs, " $2 \aleph_{0}$ " by "non denumerable."

\section{BIBLIOGRAPHY}

1. B. Dushnik and E. W. Miller, Concerning similarity transformations of linearly ordered sets, Bull. Amer. Math. Soc. vol. 46 (1940) pp. 322-326.

2. —, Partially ordered sets, Amer. J. Math. vol. 63 (1941) pp. 600-610.

3. S. Ginsburg, Some remarks on order types and decompositions of sets, Trans. Amer. Math. Soc. vol. 74 (1953) pp. 514-535.

4. - Further results on order types and decompositions of sets, Trans. Amer. Math. Soc. vol. 77 (1954) pp. 120-150.

5. - Fixed points of products and sums of simply ordered sets, Proc. Amer. Math. Soc. vol. 5 (1954) pp. 554-565.

6. H. Hahn, Reele Funktionen, New York, 1948.

7. C. Kuratowski, Topologie I, 2d. ed., Warsaw, 1948.

8. G. Kurepa, Sur les ensembles ordonnés, Hrvatske Prirodoslovno Društvo Glasnik Mat.Fiz. Astr. Ser. II vol. 3 (1948) pp. 145-151, especially p. 146.

9. W. Sierpinski, Sur une probleme concernant les sous-ensembles croissants du continu, Fund. Math. vol. 3 (1922) pp. 109-112.

10. - Exemple effectif d'une famille de $2 \aleph_{1}$ ensembles linéaires croissants, Fund. Math. vol. 35 (1948) pp. 213-216.

11. — Types d'ordre des ensembles linéaires, Fund. Math. vol. 37 (1950) pp. 253-264.

UNiversity OF Miami,

Coral Gables, Fla. 